\title{
PLANEJAMENTO FATORIAL APLICADO À DIGESTÃo DE AMOSTRAS DE FEIJÃO ASSISTIDA POR RADIAÇÃO MICROONDAS
}

Letícia M. Costa*, Maria das Graças A. Korn e Jacira T. Castro

Departamento de Química Analítica, Instituto de Química, Universidade Federal da Bahia, Campus de Ondina, 40170-290

Salvador-BA

Wagna P. C. Santos

Centro Federal de Educação Tecnológica da Bahia, Salvador-BA e Departamento de Química Analítica, Instituto de Química, Universidade Federal da Bahia, Salvador-BA

Edivan V. Carvalho

Departamento de Química, Universidade Federal de São Carlos, São Carlos- SP e Embrapa Pecuária Sudeste, São Carlos-SP

Ana Rita A. Nogueira

Embrapa Pecuária Sudeste, São Carlos-SP

Recebido em 9/9/04; aceito em 13/5/05; publicado na web em 25/11/05

\begin{abstract}
FACTORIAL DESIGN EMPLOYED FOR MICROWAVE-ASSISTED DIGESTION OF BEAN SAMPLES. The use of factorial design was evaluated for optimization of focused-microwave-assisted digestion of bean samples. Calcium, $\mathrm{Fe}, \mathrm{Mg}, \mathrm{Mn}$ and $\mathrm{Zn}$ percentual recoveries were determined in digestates after focused-microwave-assisted digestion according to factorial design procedures. A cavity microwave digestion was carried out to certify the elemental compositions obtained. The accuracy was checked using a standard reference material, the NIST SRM 8433 - Corn Bran. Results are in agreement with certified values at the $95 \%$ confidence limit when the Student $t$-test was used. Volumes of nitric and sulfuric acid, temperature, and the interplay between $\mathrm{HNO}_{3}$ and $\mathrm{H}_{2} \mathrm{SO}_{4}$ initial volumes were significant variables according to $\mathrm{P}$-values in the analysis of variance (ANOVA).
\end{abstract}

Keywords: factorial design; bean; focused-microwave-assisted digestion.

\section{INTRODUÇÃOO}

Uma das conseqüências do desenvolvimento dos métodos instrumentais, incluindo automação na geração e aquisição de dados, foi a obtenção de uma grande quantidade de dados numéricos e informações. Neste contexto, a aplicação da quimiometria facilita a interpretação de forma mais rápida e eficiente da crescente gama de dados originados de um sistema ${ }^{1}$. O planejamento fatorial é uma importante ferramenta estatística e, devido a sua simplicidade, vem sendo cada vez mais utilizada pelos químicos analíticos ${ }^{2}$ para diferentes amostras e propósitos ${ }^{3-5}$, pois possibilita a interpretação dos resultados considerando todos os parâmetros experimentais envolvidos, além de fornecer o efeito das possíveis interações entre as variáveis selecionadas.

A etapa de pré-tratamento da amostra envolve digestão, extração ou preparação do analito antes da determinação ${ }^{6}$. Essa é a etapa mais morosa de toda a análise química, requerendo cerca de $61 \%$ do tempo total necessário para emissão dos resultados, podendo ser ainda responsável por cerca de $30 \%$ do erro total da aná$\operatorname{lise}^{7}$. A utilização da radiação microondas na etapa de pré-tratamento da amostra reduz significativamente o tempo de preparo, além de contribuir para redução do branco analítico, pela utilização de menores volumes de reagentes ácidos, aumentar a segurança do operador e reduzir os riscos de perdas de elementos voláteis, quando a digestão da amostra é realizada em frascos selados, sob altas temperatura e pressão.

A associação de planejamento fatorial e procedimentos de digestão em fornos de microondas na etapa de pré-tratamento de

*e-mail: lemalta@ig.com.br amostra contribui na redução do trabalho laboratorial, além de gerar informações relevantes para mapeamento dos fatores que afetam o sistema dentro dos domínios estabelecidos.

O feijão comum (Phaseolus vulgaris L.) é uma leguminosa de grande consumo mundial, sendo o Brasil o maior produtor do gênero Phaseolus ${ }^{9}$. O feijão é uma leguminosa de grande importância nutricional, apresentando-se como fonte de minerais essenciais, tais como, $\mathrm{Ca}, \mathrm{Cu}, \mathrm{Fe}, \mathrm{K}, \mathrm{Mg}$ e $\mathrm{P}^{10}$.

Neste trabalho foi desenvolvido planejamento fatorial $2^{3}$ para otimização do programa de digestão de amostras de feijão em forno de microondas com radiação focalizada. As determinações de $\mathrm{Ca}, \mathrm{Fe}, \mathrm{Mg}, \mathrm{Mn}$ e $\mathrm{Zn}$ foram realizada por ICP OES com configuração radial. Os resultados obtidos foram comparados aos encontrados empregando um procedimento de digestão em forno de microondas com cavidade. Uma amostra de material de referência certificado, NIST SRM 8433 - Corn Bran foi utilizada para verificação da exatidão.

\section{PARTE EXPERIMENTAL}

\section{Instrumentação}

Para determinação dos analitos após digestão em forno de microondas com radiação focalizada foi utilizado um espectrômetro de emissão óptica com plasma acoplado indutivamente modelo ARL 3410 mini tocha seqüencial, com visão radial (Fisons Instruments, EUA), instalado no IQ-UFBA, Salvador-BA. Para as determinações dos elementos dos digeridos provenientes do forno de microondas com cavidade foi utilizado um espectrômetro de emissão óptica com plasma acoplado indutivamente com visão radial (Vista, Varian, Multigrave, Austrália), locado na Embrapa Pecuária 
Sudeste em São Carlos-SP. A Tabela 1 ilustra as condições experimentais utilizadas para as determinações dos analitos.

Tabela 1. Parâmetros instrumentais para as medidas por ICP OES

\begin{tabular}{lcc}
\hline & ICP OES Fisons & $\begin{array}{c}\text { ICP OES } \\
\text { Varian Vista RL }\end{array}$ \\
\hline Potência incidente (W) & 650 & 1000 \\
Câmara de nebulização & concêntrica & $\begin{array}{c}\text { ciclônica } \\
\text { Concêntrico }\end{array}$ \\
Nebulizador & 7,5 & 15 \\
Vazão do gás do plasma (L/min) & 0,81 & 1,5 \\
Vazão do gás auxiliar (L/min) & 0,81 & - \\
Vazão do fluxo carregador (L/min) & 2,5 & 0,9 \\
Vazão gás de nebulização (L/min) & 3 & 10 \\
Tempo de integração do sinal (s) & Ca I 422,67 & Ca II 317,933 \\
Linhas espectrais (nm) & Fe II 238,20 & Fe II 259,940 \\
& Mg II 279,553 & Mg II 279,800 \\
& Mn II 257,610 & Mn II 257,610 \\
& Zn I 213,856 & Zn I 213,857
\end{tabular}

I: linha atômica II: linha iônica

A digestão das amostras de feijão foi realizada no forno de microondas com radiação focalizada com duas cavidades e operando à pressão atmosférica (STAR 2, CEM, Matthews, N.C., EUA). Foi desenvolvido um procedimento para comparação e validação dos teores dos elementos estudados utilizando um forno de microondas com cavidade e capacidade para 6 frascos de perflouroalcóxi Teflon $^{\circledR}$ (PFA), equipado com sensores de pressão e temperatura (Multiwave, Anton Paar, Graz, Áustria).

As amostras de feijão foram moídas em micro-moinho de facas MA 630 (Marconi, Piracicaba, Brasil).

\section{Reagentes, soluções e amostras}

Todas as soluções foram preparadas com reagentes de grau analítico e os volumes finais ajustados com água destilada e deionizada. Todas as vidrarias foram previamente descontaminadas em banhos contendo $10 \%$ v/v $\mathrm{HNO}_{3}$ por $24 \mathrm{~h}$. Soluções de referência foram preparadas a partir de soluções estoque contendo 1000 $\mathrm{mg} \mathrm{L}^{-1}$ de $\mathrm{Ca}, \mathrm{Fe}, \mathrm{Mg}, \mathrm{Mn}, \mathrm{Zn}$ (Merck), no mesmo meio dos digeridos ácidos obtidos. Para digestão das amostras foram utilizados $\mathrm{HNO}_{3}$ e $\mathrm{H}_{2} \mathrm{SO}_{4}$ concentrados e $\mathrm{H}_{2} \mathrm{O}_{2} 30 \% \mathrm{~m} / \mathrm{v}$ (Merck).

As amostras de feijão foram adquiridas em supermercados locais e após a etapa de moagem, as porções foram homogeneizadas sendo passadas por peneira de 32 mesh e acondicionadas em sacos plásticos sob congelamento.

\section{Planejamento fatorial para digestão da amostra de feijão em forno de microondas com radiação focalizada}

Para o processo de otimização da digestão em forno de microondas com radiação focalizada foi proposto um planejamento fatorial em dois níveis $2^{3}$. O programa de aquecimento foi realizado em quatro estágios em um tempo total de $22 \mathrm{~min}$, conforme apresentado na Tabela 2. Os parâmetros avaliados foram volumes iniciais adicionados de $\mathrm{HNO}_{3}$ e $\mathrm{H}_{2} \mathrm{SO}_{4}$ concentrados e temperatura nos estágios 3 e 4, de maior incidência de radiação microondas. Para os volumes iniciais de $\mathrm{HNO}_{3}$ e $\mathrm{H}_{2} \mathrm{SO}_{4}$, os níveis inferiores e superiores relativos às variações dos parâmetros experimentais foram de 4,0 e 7,0 mL e 1,0 e 3,0 mL, respectivamente. A temperatura máxima nos estágios 3 e 4 do programa de aquecimento foi
Tabela 2. Programa de aquecimento utilizando forno de microondas com radiação focalizada (STAR 2, CEM)

\begin{tabular}{lccccc}
\multicolumn{6}{c}{ * Adição inicial de ácidos: $\mathrm{HNO}_{3}+\mathrm{H}_{2} \mathrm{SO}_{4}$} \\
\hline Etapa & $\begin{array}{c}\mathrm{t}_{\mathrm{ramp}} \\
(\mathrm{min})\end{array}$ & $\begin{array}{c}\mathrm{T} \\
\left({ }^{\circ} \mathrm{C}\right)\end{array}$ & $\begin{array}{c}\mathrm{t}_{\text {pat. }} \\
(\mathrm{min})\end{array}$ & $\begin{array}{c}\text { Reagente } \\
(\mathrm{mL})\end{array}$ & $\begin{array}{c}\text { Alíquota } \\
(\mathrm{mL})\end{array}$ \\
\hline 1 & 5 & 95 & 2 & $1,0 \mathrm{HNO}_{3}$ & 1,0 \\
2 & 3 & 150 & 2 & $1,0 \mathrm{HNO}_{3}$ & 1,0 \\
3 & 2 & $*$ & 3 & - & - \\
4 & - & $*$ & 5 & $10,0 \mathrm{H}_{2} \mathrm{O}_{2}$ & 1,0 \\
\hline
\end{tabular}

* Parâmetros variados durante execução do planejamento fatorial (volume inicial de $\mathrm{HNO}_{3}$ variando de 4,0 a 7,0 mL, volume inicial $\mathrm{H}_{2} \mathrm{SO}_{4}$ variando de 1,0 a $3,0 \mathrm{~mL}$ e temperatura nos estágios 3 e 4 variando de 170 a $200{ }^{\circ} \mathrm{C}$ ).

selecionada para $170{ }^{\circ} \mathrm{C}$, no nível inferior e $200{ }^{\circ} \mathrm{C}$ no nível superior. O ponto central das variáveis foi obtido pela média dos valores inferiores e superiores de cada parâmetro. Foram digeridas cerca de $500 \mathrm{mg}$ de amostra e as soluções límpidas dos digeridos, transferidas para balão volumétrico de $25 \mathrm{~mL}$. Todos os experimentos foram desenvolvidos em duplicata, de forma randômica, e no ponto central foram realizados experimentos em quadruplicata. Os dados experimentais foram processados empregando-se o programa Statistica (Statistica for Windows, Statsoft, Inc, 1999. Tulsa, OK, EUA).

\section{Procedimentos de digestão em forno de microondas com cavidade}

O programa de aquecimento utilizado para digestão das amostras de feijão em forno de microondas com cavidade está apresentado na Tabela 3. Para uma massa de cerca de $200 \mathrm{mg}$ de amostra, pesada diretamente nos frascos reacionais, adicionou-se $2,0 \mathrm{~mL}$ de $\mathrm{HNO}_{3}$ concentrado e $1,0 \mathrm{~mL}$ de $\mathrm{H}_{2} \mathrm{O}_{2} 30 \% \mathrm{~m} / \mathrm{v}$. Após resfriamento, os digeridos foram diluídos para $10 \mathrm{~mL}$ com água deionizada.

Tabela 3. Programa de aquecimento do forno de microondas com cavidade (Multiwave) e frascos fechados (temperatura máxima $=$ $\left.190{ }^{\circ} \mathrm{C}\right)$

\begin{tabular}{lccccl}
\hline Etapa & $\begin{array}{c}\text { Potência } \\
\text { inicial (W) }\end{array}$ & $\begin{array}{c}\text { Tempo } \\
(\mathrm{min})\end{array}$ & $\begin{array}{r}\text { Potência } \\
\text { final (W) }\end{array}$ & $\begin{array}{c}\text { Ventilação } \\
(\%)\end{array}$ & Descrição \\
\hline 1 & 400 & 3 & 400 & 25 & Pré-aquecimento \\
2 & 850 & 6 & 850 & 25 & Aquecimento \\
3 & 1000 & 10 & 1000 & 25 & Aquecimento \\
4 & 0 & 15 & 0 & 100 & Resfriamento \\
\hline
\end{tabular}

\section{RESULTADOS E DISCUSSÃO}

\section{Planejamento fatorial}

Os parâmetros experimentais selecionados para o planejamento fatorial $2^{3}$ aplicado ao procedimento de digestão em forno de microondas com radiação focalizada para as amostras de feijão foram volume inicial de $\mathrm{HNO}_{3}$, volume inicial de $\mathrm{H}_{2} \mathrm{SO}_{4}$ e temperatura da etapa de maior incidência de radiação microondas. $\mathrm{O}$ planejamento fatorial foi desenvolvido nos níveis máximo e mínimo e o percentual de concordância de $\mathrm{Ca}, \mathrm{Fe}, \mathrm{Mg}, \mathrm{Mn}$ e $\mathrm{Zn}$ foi a resposta avaliada após a execução dos experimentos, como ilustra a Tabela 5. A significância dos efeitos das variáveis e das possíveis 
interações entre elas foi checada pela aplicação da análise de variância (ANOVA) e com base nos gráficos de Pareto (Figura 1). As informações obtidas após a construção de superfícies de resposta, como ilustrado na Figura 2, também foram utilizadas na interpretação dos resultados.

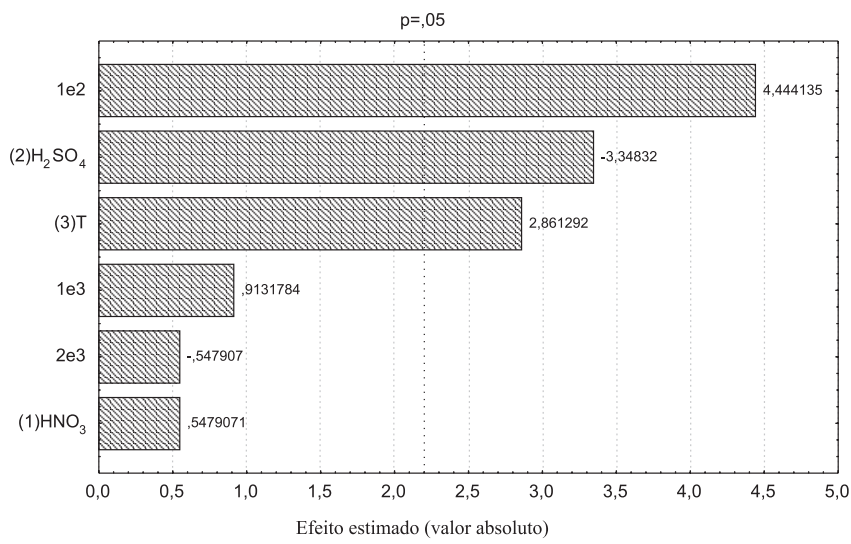

Figura 1. Gráfico de Pareto do planejamento fatorial completo $2^{3}$ obtido para Fe (a linha vertical define 95\% do intervalo de confiança)

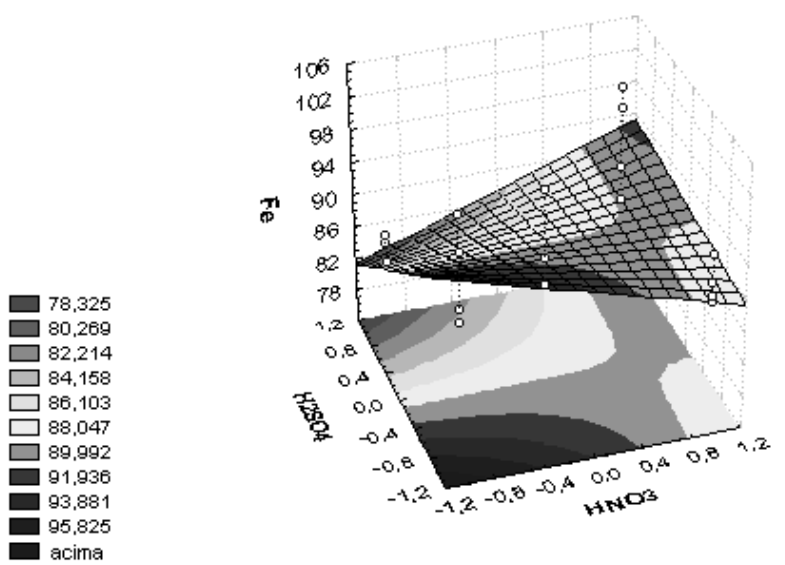

Figura 2. Superfície de resposta do planejamento fatorial completo $2^{3}$ obtido para $\mathrm{Fe}$ (-1,2: nível mínimo; 1,2: nível máximo; 0: ponto central)

Os valores de concentrações dos elementos $\mathrm{Ca}, \mathrm{Fe}, \mathrm{Mg}, \mathrm{Mn}$ e $\mathrm{Zn}$ encontrados nas amostras de feijão digeridas no forno de microondas com cavidade foram de $833 \pm 27 ; 49,0 \pm 1,0 ; 1839 \pm 15$; $18,7 \pm 0,3 ; 37,8 \pm 0,8 \mathrm{mg} \mathrm{g}^{-1}$, respectivamente. Os gráficos de Pareto e as superfícies de resposta foram construídos a partir dos percentuais de recuperação dos elementos após aplicação do planejamento fatorial $2^{3}$ e tomando-se como referência os valores determinados após digestão das amostras em forno de microondas com cavidade, sob altas temperatura e pressão. Segundo a análise de variância e pelos gráficos de Pareto, tem-se que a variável volume inicial de $\mathrm{H}_{2} \mathrm{SO}_{4}$ foi significativa para todos os elementos avaliados e com efeito negativo, isto é, para menores volumes de $\mathrm{H}_{2} \mathrm{SO}_{4}$ foram obtidos maiores percentuais de concordância dos elementos, sendo que, para $\mathrm{Zn}$ e Ca essa foi a única variável significativa.

A interação entre as variáveis volume de $\mathrm{HNO}_{3}$ e de $\mathrm{H}_{2} \mathrm{SO}_{4}$ foi significativa para $\mathrm{Mg}, \mathrm{Mn}$ e $\mathrm{Fe}$, com um valor positivo associado a ela. Isto significa que um aumento no volume da mistura ácida oxidante com maior proporção de $\mathrm{HNO}_{3}$ em relação ao $\mathrm{H}_{2} \mathrm{SO}_{4}$ pode levar a maiores percentuais de recuperação para esses elementos.

Para o Fe (Figura 1) a temperatura, a interação entre as variá- veis volume de $\mathrm{HNO}_{3}$ e de $\mathrm{H}_{2} \mathrm{SO}_{4}$ e o volume de $\mathrm{H}_{2} \mathrm{SO}_{4}$ foram as variáveis significativas. A variável temperatura foi significativa somente para $\mathrm{Fe}$, com um valor positivo associado, ou seja, maior recuperação é obtida com o aumento da temperatura da mistura digestora. Para Ca e $\mathrm{Zn}$, teores de recuperação em torno de $70 \%$ foram encontrados nos digeridos do forno de microondas com radiação focalizada, quando comparados aos valores obtidos pelo sistema de microondas com cavidade. A formação de sulfatos pouco solúveis e a perda por volatilização, no caso do $\mathrm{Zn}$, podem explicar essa tendência. Possivelmente, esses teores seriam superiores se houvesse a possibilidade de realização da digestão no sistema focalizado sem adição do ácido sulfúrico. Porém, como o sistema opera à pressão atmosférica, a utilização de $\mathrm{H}_{2} \mathrm{SO}_{4}$ é necessária para alcançar temperaturas superiores à temperatura de ebulição do $\mathrm{HNO}_{3}\left(120^{\circ} \mathrm{C}\right)$ e promover uma eficiente oxidação da matéria orgânica sem que ocorra a secura da mistura digestora evitando, assim, acidentes e/ou danos ao equipamento. Entretanto, sempre que possível, a utilização de menores volumes de $\mathrm{H}_{2} \mathrm{SO}_{4}$ em programas de digestão de amostras pode ser vantajosa, pois a baixa acidez dos digeridos propicia menores fatores de diluição, possibilitando introdução direta em equipamentos, ampliando a possibilidade de detecção dos elementos presentes e aumentando a vida útil dos dispositivos ópticos e eletrônicos que sofrem ação direta das soluções ácidas ${ }^{11}$.

\section{Validação dos resultados}

Para a amostra certificada (NIST SRM 8433), os resultados após a digestão em forno com cavidade são apresentados na Tabela 4. Os valores encontrados concordam com os valores certificados a um nível de confiança de $95 \%$, empregando-se o teste $t$-Student pareado.

Tabela 4. Valores certificados e determinados de $\mathrm{Ca}, \mathrm{Fe}, \mathrm{Mg}, \mathrm{Mn}$ e Zn para a amostra NIST SRM 8433 - Corn Bran, digerida em forno de microondas com cavidade

\begin{tabular}{lcc}
\hline Elemento & Valor certificado & Valor encontrado \\
\hline $\mathrm{Ca}$ & $420 \pm 38$ & $415 \pm 5$ \\
$\mathrm{Mg}$ & $818 \pm 59$ & $833 \pm 12$ \\
$\mathrm{Mn}$ & $2,55 \pm 0,2$ & $2,4 \pm 0,1$ \\
$\mathrm{Zn}$ & $18,6 \pm 2,2$ & $16,9 \pm 0,3$ \\
\hline
\end{tabular}

Os valores dos percentuais de recuperação de $\mathrm{Ca}, \mathrm{Fe}, \mathrm{Mg}, \mathrm{Mn}$ e $\mathrm{Zn}$ obtidos após o planejamento experimental, tomando como valores de referência o procedimento de digestão em forno de microondas com cavidade sob altas temperatura e pressão, são mostrados na Tabela 5. O procedimento de digestão em forno de microondas sob altas temperatura e pressão foi selecionado como método de referência por apresentar menor susceptibilidade a perdas por volatilização e contaminação, o que resulta em baixos valores de brancos analíticos. Os resultados apresentados na Tabela 5 indicam uma faixa de recuperação variando de 74 a $90 \%$ para Ca, 78 a $104 \%$ para $\mathrm{Fe}, 78$ a $91 \%$ para $\mathrm{Mg}, 74$ a $88 \%$ para $\mathrm{Mn}$ e 65 a $84 \%$ para $\mathrm{Zn}$, quando comparados aos valores obtidos após digestão em forno de microondas com cavidade. Os melhores percentuais de recuperação dos elementos foram obtidos nas condições de menores volumes $\mathrm{H}_{2} \mathrm{SO}_{4}$. Os resultados alcançados após a digestão em forno de microondas com radiação focalizada concordam com os valores obtidos no forno de microondas com cavidade a um nível de confiança de $95 \%$, empregando-se o teste $t$-Student. 
Tabela 5. Porcentagem de recuperação de $\mathrm{Ca}, \mathrm{Fe}, \mathrm{Mg}, \mathrm{Mn}$ e $\mathrm{Zn}$ após realização do planejamento fatorial $2^{3}$

\begin{tabular}{ccccccccc}
\hline EXP. & $\mathrm{HNO}_{3}$ & $\mathrm{H}_{2} \mathrm{SO}_{4}$ & $\mathrm{~T}$ & \multicolumn{5}{c}{ RECUPERAÇ̃ } \\
& & & & $\mathrm{Zn}$ & $\mathrm{Fe}$ & $\mathrm{Mn}$ & $\mathrm{Mg}$ & $\mathrm{Ca}$ \\
\hline 1 & - & - & - & 84 & 91 & 85 & 91 & 90 \\
2 & + & - & - & 83 & 85 & 81 & 85 & 86 \\
3 & - & + & - & 70 & 81 & 74 & 78 & 77 \\
4 & + & + & - & 75 & 81 & 75 & 78 & 76 \\
5 & - & - & + & 84 & 104 & 88 & 90 & 85 \\
6 & + & - & + & 78 & 92 & 81 & 85 & 85 \\
7 & - & + & + & 73 & 78 & 78 & 84 & 80 \\
8 & + & + & + & 74 & 98 & 81 & 81 & 78 \\
9 & 0 & 0 & 0 & 77 & 95 & 82 & 86 & 84 \\
10 & 0 & 0 & 0 & 75 & 81 & 75 & 82 & 74 \\
11 & - & - & - & 83 & 89 & 84 & 87 & 87 \\
12 & + & - & - & 71 & 88 & 85 & 83 & 83 \\
13 & - & + & - & 69 & 82 & 74 & 80 & 78 \\
14 & + & + & - & 78 & 86 & 84 & 82 & 82 \\
15 & - & - & + & 81 & 99 & 85 & 88 & 84 \\
16 & + & - & + & 80 & 86 & 84 & 85 & 87 \\
17 & - & + & + & 76 & 78 & 74 & 82 & 85 \\
18 & + & + & + & 79 & 95 & 80 & 82 & 80 \\
19 & 0 & 0 & 0 & 80 & 85 & 81 & 85 & 81 \\
20 & 0 & 0 & 0 & 79 & 81 & 78 & 83 & 80 \\
\hline
\end{tabular}

\section{CONCLUSÃO}

A utilização do planejamento fatorial indicou uma tendência para obtenção de resultados com melhores percentuais de recuperação. A variável volume inicial de $\mathrm{H}_{2} \mathrm{SO}_{4}$ foi significativa para todos os elementos investigados. Porém, sua utilização em sistemas que operam à pressão atmosférica é necessária para o aumento da temperatura acima do ponto de ebulição do ácido nítrico $\left(120{ }^{\circ} \mathrm{C}\right)$, mas sua redução deve ser avaliada, pois permite a obtenção de melhores percentuais de concordância para a amostra em estudo. A interação das variáveis volume de $\mathrm{HNO}_{3}$ e de $\mathrm{H}_{2} \mathrm{SO}_{4}$ aponta para um aumento no volume da mistura ácida oxidante para obten- ção de melhores recuperações para Fe, Mg e Mn. Neste caso, a redução do $\mathrm{H}_{2} \mathrm{SO}_{4}$ e concomitante elevação do volume de $\mathrm{HNO}_{3}$ pode gerar condições reacionais favoráveis à digestão da matéria orgânica, com melhores recuperações desses elementos. O procedimento de digestão empregado na digestão da amostra de material de referência certificado gerou resultados precisos e exatos. Os resultados evidenciam uma das principais vantagens da utilização do planejamento fatorial: a possibilidade de avaliar o sistema de maneira multivariada, buscando otimizar todas as variáveis que compõem o sistema experimental.

\section{AGRADECIMENTOS}

À Fundação de Amparo à Pesquisa do Estado da Bahia FAPESB (PRONEX-CNPq-FAPESB - Processo número 40/03), CAPES e FINEP pelo suporte financeiro. Os autores L. M. Costa, E. V. Carvalho, M. G. A. Korn, A. R. A. Nogueira e J. T. Castro agradecem ao $\mathrm{CNPq}$ pelas bolsas concedidas.

\section{REFERÊNCIAS}

1. Pereira-Filho, E. R.; Poppi, R. J.; Arruda, M. A. Z.; Quim. Nova 2002, 25, 246.

2. Neto, B. B.; Scarminio, I. S.; Bruns, R. E.; Como fazer experimentos pesquisa e desenvolvimento na ciência e na indústria, Ed. Unicamp: Campinas, 2001.

3. Zhou, C. Y.; Wong, M. K.; Koh, L. L.; Wee, Y. C.; Anal. Chim. Acta 1995, 314, 121.

4. Martín-Garcia, M. B.; Bellido-Milla, D.; Jimenez-Jimenez, A.; HernándezArtiga, M. P.; Fresenius J. Anal. Chem. 1999, 364, 527.

5. Ferreira, S. L. C.; dos Santos, W. N. L.; Bezerra, M. A.; Lemos, V. A.; Bosque-Sendra, J. M.; Anal. Bioanal. Chem. 2003, 375, 443.

6. Oliveira, E.; J. Braz. Chem. Soc. 2003, 14, 174.

7. Arruda, M. A. Z.; Santelli, R. H.; Quim. Nova 1997, 20, 638.

8. Krug, F. J.; Métodos de decomposição de amostras, III Workshop sobre preparo de amostras, FAPESP, São Carlos, 2000.

9. Yokoyama, L. P. Em Cultura do feijoeiro no Brasil: características da produção, Yokoyama, L. P.; Stone L. F., eds.; Embrapa Arroz e Feijão, 2000.

10. Canniatti-Brazaca, S. G.; Novaes, N. J.; Salgado, J. M.; Marques, U. M. L.; Mancini-Filho, J.; Ciênc. Tecnol. Aliment. 1996, 16, 36.

11. Todolí, J. L.; Mermet, J. M.; Spetrochim. Acta 1999, 54B, 895. 